\title{
Influence of intermolecular interactions on the properties of carbon nanotubes
}

\author{
PIOTR KAMEDULSKI ${ }^{1, *}$, ANNA KACZMAREK-KEDZIERA ${ }^{1}$ and JERZY P LUKASZEWICZ ${ }^{1,2}$ \\ ${ }^{1}$ Faculty of Chemistry, Nicolaus Copernicus University, 87-100 Toruń, Poland \\ ${ }^{2}$ Centre for Modern Interdisciplinary Technologies, Nicolaus Copernicus University, 87-100 Torun, Poland \\ *Author for correspondence (piotrkam@doktorant.umk.pl)
}

MS received 14 August 2017; accepted 7 November 2017; published online 19 May 2018

\begin{abstract}
This study is aiming at theoretical and experimental confirmations of the deposition of oligothiophene dyes (OT) on structurally and chemically diverse surfaces of carbon materials, especially carbon nanotubes (CNTs). This study is a contribution to the wide search and design of novel hybrid materials for light-sensitive (dye solar cells) and light-emitting (LEDs) structures. The obtained materials containing OTs embedded in the pores of a carbon matrix were analysed by means of microscopic and spectroscopic techniques as well as low-temperature adsorption of nitrogen, together with an investigation of the surface area by the BET method. Confocal microscopy was employed to confirm the immobilization of oligomers on the surface of CNTs. Raman spectra, XRD and HTEM microscopies allowed to judge the quality of the carbonaceous material and to compare the test material before and after the introduction of OT. Additionally, the elemental content and elemental surface species were determined by means of XPS and combustion elemental analyses. The explanation of thiophene; $2,2^{\prime}: 5^{\prime}, 2^{\prime \prime}$-terthiophene and $\alpha$-sexithiophene molecular interactions with single wall armchair CNTs was supported by theoretical calculations. In computational investigations, CNTs were modelled by tubules of different sizes, lengths and hydrogenated open ends.
\end{abstract}

Keywords. Oligothiophenes (OTs); carbon nanotubes (CNTs); organic dyes; confocal microscopy; theoretical calculations; interaction energy.

\section{Introduction}

One of the most emerging goals of contemporary material science is to obtain carbon-based materials, which have unique and potentially applicable optical properties. Such research attempts belong to widely understood organic electronics i.e., an emerging branch of contemporary technology. A very important advantage of organic electronics is a possibility of precise adjustment in the electronic and optoelectronic characteristics of organic semiconductors like carbon-based materials. It is expected that in the near future, organic semiconductors will lead to the creation of lowcost, lightweight and high-performance electronic devices. The wide range of fascinating properties of these nanoscale molecules provides attractive opportunities for technological applications.

Non-covalent functionalization of such materials may be an efficient tool for tuning their electronic and optical properties [1-3]. There are two main strategies for non-covalent functionalization of carbon nanotubes (CNTs). The first one is to change the outer surface of CNTs by adsorption of organic molecules. This strategy is relatively easy, as there are no obvious restrictions to the size, position and arrangement of the attached molecule. The second possibility is to encapsulate organic molecules inside the carbon nanotube. Encapsulation of organic molecules does not lead to structural changes, thus, maintaining the desired properties of carbon material. In particular, functionalization increases the chemical reactivity, which in turn enhances interactions between the small organic molecule and the nanotube.

Currently, the development of different methodologies and discovering new materials that can modify the optical properties of CNTs is clearly required. Encapsulation of different organic molecules inside CNTs was reported in the literature during the last 10 years [4-7]. The research groups of Simon and Yudasaka studied properties of SWCNT/ $\mathrm{C}_{60} \mathrm{com}-$ posites and the efficiency in releasing of $\mathrm{C}_{60}$ from SWCNT $[6,7]$. It was shown that fullerenes could be removed from the inside of SWCNTs with high efficiency using dichlorobenzene as a solvent. Other types of investigated guest molecules are organic substances, such as L-histidine [8], $\beta$-carotene or squarylium (SQ) dye [9], which can be encapsulated inside the nanotubes. 
Conjugated oligomers and polymers based on heterocyclic compounds have attracted strong interest in recent years due to their potential applications in organic electronic devices, such as photovoltaic cells, organic light-emitting diodes (OLEDs) and organic field-effect transistors (OFETs) [5]. One of such promising materials are oligothiophenes (OTs). OT dyes exhibit unique optical and electronic properties, which can be fine-tuned through small structural modifications. Nowadays, they are one of the most studied organic semiconductor materials [10-12].

Loi et al [4] first reported the synthesis of OTs inside a nanotube and confirmed the encapsulation process using different experimental methods, such as high-resolution transmission electron microscopy (HR-TEM) and Raman spectroscopy. In turn, Gidron et al [13] described the new synthesis of two structural isomers containing a bifuran or a bithiophene unit, which have the advantages of both oligoheterocyclic compounds. Recently, Gao et al [14] reported on the methods for selective separation of semiconducting SWCNTs from the commercial nanotubes using OTs dyes. These materials are considered to be promising low-cost alternatives to the traditional electronic devices based on typical inorganic semiconductors, due to their remarkable electronic and optical properties. Many OT dyes exhibit the possibility of forming dimers or larger aggregates [15-17]. However, their deposition on a solid substrate is inevitable in many potential applications. This concept was not widely exploited yet and still has a high level of novelty. CNTs may serve as such a platform, since they are very stable, flexible and used as electrically conductive materials $[18,19]$. Despite these useful properties, one should keep in mind that CNTs also have a handful of drawbacks, such as low solubility in various solvents or wide range of light absorption. This fact may rise manufacturing obstacles, which must be overcome. Therefore, the work on improving the CNTs characteristics is still ongoing.

The aim of the study was to obtain carbon materials based on CNTs modified with OTs and their characterization in particular to investigate the properties of the nanotube hybrid systems and to study the nature of the interaction of selected OT dyes with nanotubes. These materials were chosen for several reasons. Firstly, the OT dye molecules are small enough to be encapsulated into CNTs. Secondly, OTs have a well proven high chemical and environmental stability, which in combination with CNTs, could constitute an attractive object of study for applications in various industries, especially electronics.

In this work, a detailed microscopic and spectroscopic analyses of the synthesized complexes (oligothiopene dyesCNTs) are applied to indicate the most beneficial way of nanotube modification by OT dyes in terms of their potential applications. The recent studies on this class of compounds/materials were predominantly focussed on their structure and intermolecular interactions [4,9,20]. Similar investigations targeted at deposited dyes are rare. Therefore, based on the gathered information, the authors assume that further studies seem necessary to generalize the observations.

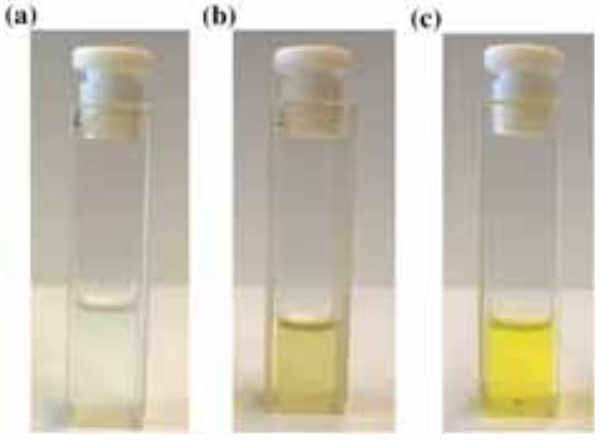

Figure 1. Dye solution in chloroform for (a) $\mathrm{T}$; (b) $3 \mathrm{~T}$ and (c) $6 \mathrm{~T}$.

\section{Experimental}

\subsection{Materials}

All organic dyes were purchased from Sigma-Aldrich: thiophene (CAS number: 110-02-1), 2,2':5',2'-terthiophene (CAS number: 1081-34-1) and $\alpha$-sexithiophene (CAS number: 88493-55-4). Two kinds of MWCNTs with differing diameters were purchased, processed and applied in this study. MWCNTs were bought as commercial products from SkySpring nanomaterials. The parameters of the declared MWCNTs (with larger diameters) are as follows: both ends closed, purity: $>95 \%$, outer diameter 10-20 nm, inner diameter 3-5 nm. Whereas MWCNTs (with smaller diameters) were characterized as: both ends closed, purity $90 \%$, outer diameter 1-2 nm; inner diameter 0.8-1.6 nm. Both kinds of MWCNTs were further purified and opened. After purification and opening, CNTs were dispersed into the dye solution in chloroform to adsorb the dye molecules or encapsulate them inside the material. The surface area of the resulting complexes was analysed to determine the degree of dye adsorption. Further in the text, the dye solutions in chloroform are called T (thio-

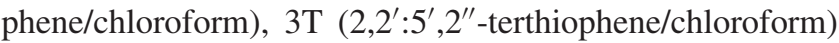
and 6T ( $\alpha$-sexithiophene/chloroform), respectively, which are shown in figure 1.

A complex and thorough analysis was performed to determine the surface structure, chemical composition, surface area and optical properties of the purified carbon materials and the obtained CNTs/dye complexes.

\subsection{Method of preparation of CNTs}

MWCNTs were functionalized by liquid oxidation using $\mathrm{KMnO}_{4}$ to obtain the reactive groups on their surface and their opening for encapsulating agent. Twenty-one grams of $\mathrm{KMnO}_{4}$ were diluted in $250 \mathrm{ml}$ of $1 \mathrm{M} \mathrm{H}_{2} \mathrm{SO}_{4}$. Four grams of as-delivered MWCNTs (4.1 g MWCNTs with smaller diameters) were added to the solution. The mixture was boiled at $80^{\circ} \mathrm{C}$ for $1 \mathrm{~h}$ in the water bath. MWCNTs were washed with water $(5 \times 300 \mathrm{ml})$, filtered through a Büchner funnel 
and dried for $4 \mathrm{~h}$ at $80^{\circ} \mathrm{C}$. This process can be described as:

$$
\begin{aligned}
& 3 \mathrm{C}+4 \mathrm{KMnO}_{4}+4 \mathrm{H}^{+} \rightarrow 4 \mathrm{MnO}_{2}+3 \mathrm{CO}_{2} \\
& +4 \mathrm{~K}^{+}+2 \mathrm{H}_{2} \mathrm{O} .
\end{aligned}
$$

The mass of the product, which is much greater than the mass of the substrate, indicates the presence of a large amount of a byproduct, which was identified as $\mathrm{MnO}_{2}$. Due to the presence of $\mathrm{MnO}_{2}$, further reactions with $\mathrm{HCl}$ were carried out to remove it. The reaction with $\mathrm{HCl}$ was carried out in the following way. Firstly, 116,265 g of MWCNTs (previously oxidized by $\mathrm{KMnO}_{4}$ ) were added to $600 \mathrm{ml}$ of $2 \mathrm{M} \mathrm{HCl}$ solution. The beaker with the mixture was inserted into a water bath and boiled at $80^{\circ} \mathrm{C}$ for $1 \mathrm{~h}$. Then, MWCNTs were washed with distilled water $(5 \times 300 \mathrm{ml})$, filtered through a Büchner funnel until the neutral $\mathrm{pH}$ was reached. Next, the rinsed carbons were dried for $4 \mathrm{~h}$ at $80^{\circ} \mathrm{C}$. The reaction with $\mathrm{HCl}$ can be described as:

$$
4 \mathrm{HCl}+\mathrm{MnO}_{2} \rightarrow \mathrm{MnCl}_{2}+\mathrm{Cl}_{2}+2 \mathrm{H}_{2} \mathrm{O} .
$$

A similar set of reactions was performed in the case of MWCNTs with smaller diameters.

Next, $25 \mathrm{ml}$ of organic dye solution in chloroform (4 $\left.\times 10^{-3} \mathrm{M}\right)$ was prepared. The solution was quantitatively transferred into the $100 \mathrm{ml}$ volumetric flask, filled to the mark with chloroform and shaken. The encapsulation of the dyes (T, 3T, 6T) in open MWCNTs started after the addition of $0.3 \mathrm{~g}$ of CNTs to each flask. The dye adsorption process lasted for 4 days. The mixtures were shaken every 3-4 h. The obtained materials were denoted as T@MWCNTs_ld,3T@MWCNTs_ld,6T@MWCNTs_ld (@ symbol suggests that the chain molecules are encapsulated in the nanotubes.) for nanotubes with larger diameters and T@MWCNTs_sd, 3T@MWCNTs_sd, 6T@MWCNTs_sd for tubes with smaller diameters. The materials were washed with chloroform $(2 \times 100 \mathrm{ml})$, filtered through a Büchner funnel and dried in an oven for $4 \mathrm{~h}$ at $80^{\circ} \mathrm{C}$. Additional information on the applied procedure can be found in the supporting information (table S1).

\subsection{Elemental analysis}

The volumetric elemental composition (carbon, nitrogen, hydrogen and total residue elements) of the materials was analysed by means of a combustion elemental analyzer (Vario MACRO CHN, Elementar Analysensysteme GmbH).

\subsection{Surface area and pore structure}

The structural parameters of the CNTs were characterized by low-temperature adsorption of nitrogen method. Before each adsorption measurement, the sample was outgassed under vacuum at $200^{\circ} \mathrm{C}$ for $2 \mathrm{~h}$. The relevant isotherms of all the samples were measured at $77 \mathrm{~K}$ on a Gemini VI volumetric adsorption analyzer (Micromeritics, USA). A specific surface area was determined by the Brunauer-Emmett-Teller (BET) method $\left(S_{\mathrm{BET}}\right)$.

\subsection{SEM/EDX analysis}

The CNTs were examined by scanning electron microscopy (SEM, 1430 VP, LEO Electron Microscopy Ltd.) with an energy dispersive X-ray spectrometer (EDX, Quantax 200; detector: XFlash 4010, Bruker AXS). Then, the results of the three areas are averaged.

\subsection{HR-TEM analysis}

The CNTs were examined by HR-TEM (FEI Europe production, model Tecnai F20 X-Twin). The nanotubes were dispersed in chloroform and treated with Inter Sonic IS-1K bath (95 W for $15 \mathrm{~min}$ ) and dropped onto porous carboncoated copper grids.

\subsection{Confocal microscopy}

The CNTs were examined by confocal microscopy (model: Leica TCS SPE). In fluorescence investigation, laser beams of wavelengths 405, 561 and $633 \mathrm{~nm}$ were used. Four lasers were turned on at the same time: Diode $(405 \mathrm{~nm}), 3 \%$ Argon, DPSS $(561 \mathrm{~nm})$ and linear-polarized He-Ne laser $(633 \mathrm{~nm})$. The confocal image was obtained using a single excitation at $405 \mathrm{~nm}$ (UV lamp) and second excitation at $458 \mathrm{~nm}(5 \%)$ (Zoom x1, Pinhol x1, 63x Objective). The sample was placed on a microscope slide with a coverslip. All confocal microscopy measurements are carried out in ambient conditions.

\subsection{Raman spectroscopy}

The CNTs were examined by Raman spectroscopy (microscop Renishaw InVia, Renishaw plc (Gloucestershire, UK), Laser: Modu-Laser Stellar-REN, Multi-Line (max. Power 150 $\mathrm{mW}$ ), Leica DM1300M camera Infinity 1; objective: Leica, N PLAN L50x/0.5). All the spectra were collected at ambient temperature.

\section{$2.9 X P S$}

The chemical structure of CNTs and the elemental composition of their surface were determined using X-ray photoelectron spectroscopy (XPS) analysis. The photoelectron spectra were studied by means of an ESCALAB-210 (VG Scientific) spectrometer using $\mathrm{AlK} \alpha(1486.6 \mathrm{eV})$ from an X-ray source operating at $14.5 \mathrm{kV}$ and $25 \mathrm{~mA}$. Survey spectra were recorded for all the samples in the energy range from 0 to $1350 \mathrm{eV}$ with $0.4 \mathrm{eV}$ step. High-resolution spectra were recorded with $0.1 \mathrm{eV}$ step, $100 \mathrm{~ms}$ dwell time and $25 \mathrm{eV}$ pass energy. A $90^{\circ}$ take-off angle was used in all the measurements. The curve fitting was performed using the AVANTAGE software provided 

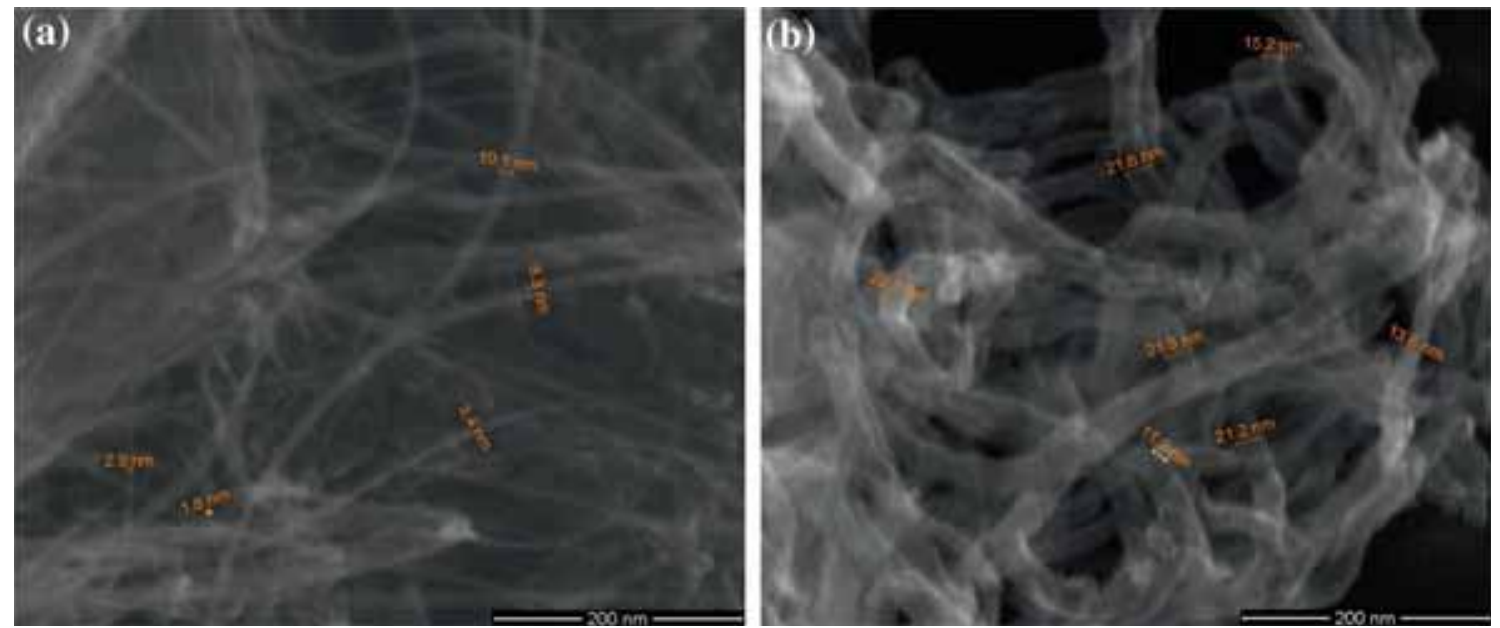

Figure 2. SEM images of (a) MWCNT_sd and (b) MWCNT_ld with marked diameters.

by Thermo Electron, which describes each component of the complex envelope as a Gaussian-Lorentzian sum function; a constant $0.3( \pm 0.05) \mathrm{G} / \mathrm{L}$ ratio was used. The background was fitted using nonlinear Shirley model. Scofield sensitivity factors and measured transmission function were used for quantification.

\section{$2.10 X R D$ analysis}

The CNTs were examined by X-ray diffraction (XRD) method to identify the presence of non-filled CNTs as well as CNTs with encapsulated/adsorbed oligoheterocyclic dyes. The wide-angle XRD patterns were recorded on a X'Pert Philips difractometer using $\mathrm{CuK} \alpha$ radiation $(\alpha=1.5406 \AA)$, (40 kV and $30 \mathrm{~mA}$ conditions). The experiments were performed over the $2 \theta$ range of $5-120^{\circ}$ where divergence slit size was $0.0167^{\circ}$ and scan step time was $20 \mathrm{~s}$.

\subsection{Computational details}

The geometry optimization of the isolated OT molecules and CNT were performed with B97-D/6-31G(d,p) approach. The calculations of the nanotubes containing thiophene oligomers involved up to 6564 basis functions. Three density functionals B97-D; B97-D3 and wB97x-D with the 6-31G(d,p) basis set are applied to investigate the interactions of $(7,7)$ SWCNTs with OTs dyes. Nanotubes in the armchair configuration with both ends saturated with hydrogen atoms were investigated in the calculations. The interaction energy was estimated with a supermolecular approach with counterpoise correction included. All quantum chemical calculations were performed using the Gaussian 09 software package $[21,22]$.

\section{Results and discussion}

Various novel analytical techniques were applied for the study of these nanomaterials and the obtained results are discussed
Table 1. Elemental content of $\mathrm{N}, \mathrm{C}$ and $\mathrm{H}$ in the MWCNT_ld before and after purification/opening treatments.

\begin{tabular}{lccc}
\hline MWCNT_ld & N & C & H \\
\hline Before purification/opening treatment & 0.271 & 96.43 & 0.220 \\
After $\mathrm{KMnO}_{4}$ treatment & 0.044 & 18.41 & 0.729 \\
After $\mathrm{HCl}$ treatment & 0.140 & 89.49 & 0.519 \\
\hline
\end{tabular}

in this article. Figure 2 shows SEM images of investigated nanotubes. For MWCNT_sd samples, diameter ranges from 1.8 to $10.1 \mathrm{~nm}$, while for MWCNT_ld samples, the diameter ranges from 11.2 to $22.5 \mathrm{~nm}$.

The combustion elemental analysis of the CNTs before encapsulation was performed to evaluate the purity of CNTs after opening. The results for MWCNT_ld sample are presented in table 1 .

The observed loss of carbon content $(\sim 7.2 \%)$ after all the purification/opening treatments is relatively small, but not negligible. It is attributed to the partial oxidation of CNTs, which is a side effect of CNTs opening by means of oxidative methods [23]. Despite its exactness, the combustion elemental analysis delivers a limited amount of information, i.e., only three elements are detectable (hydrogen, nitrogen and carbon). Therefore, the oxygen content in CNTs could not be determined in this experiment. To confirm the presence of the oxygen content, the EDX analysis was carried out, which allows to learn about the composition of the other elements in CNTs (tables 3, 4 and 5).

BET surface area was measured for both types of CNTs before and after the encapsulation/adsorption of OT dyes. On the basis of changes in the surface area, the degree of encapsulation of the OTs can be specified. Results of the BET surface area of dye-modified CNTs obtained by means of lowtemperature nitrogen adsorption are presented in table 2.

Pristine (opened and purified) materials reach a surface area of $278 \mathrm{~m}^{2} \mathrm{~g}^{-1}$ for MWCNT with small diameters and 
Table 2. Specific surface area, $S_{\mathrm{BET}}$ of investigated materials.

\begin{tabular}{lccc}
\hline Sample & $S_{\text {BET }}\left(\mathrm{m}^{2} \mathrm{~g}^{-1}\right)$ & Sample & $S_{\text {BET }}\left(\mathrm{m}^{2} \mathrm{~g}^{-1}\right)$ \\
\hline MWCNT_sd & 278 & MWCNT_ld & 249 \\
T@MWCNT_sd & 207 & T@MWCNT_ld & 249 \\
3T@MWCNT_sd & 210 & 3T@MWCNT_ld & 244 \\
6T@MWCNT_sd & 174 & 6T@MWCNT_ld & 228 \\
\hline
\end{tabular}

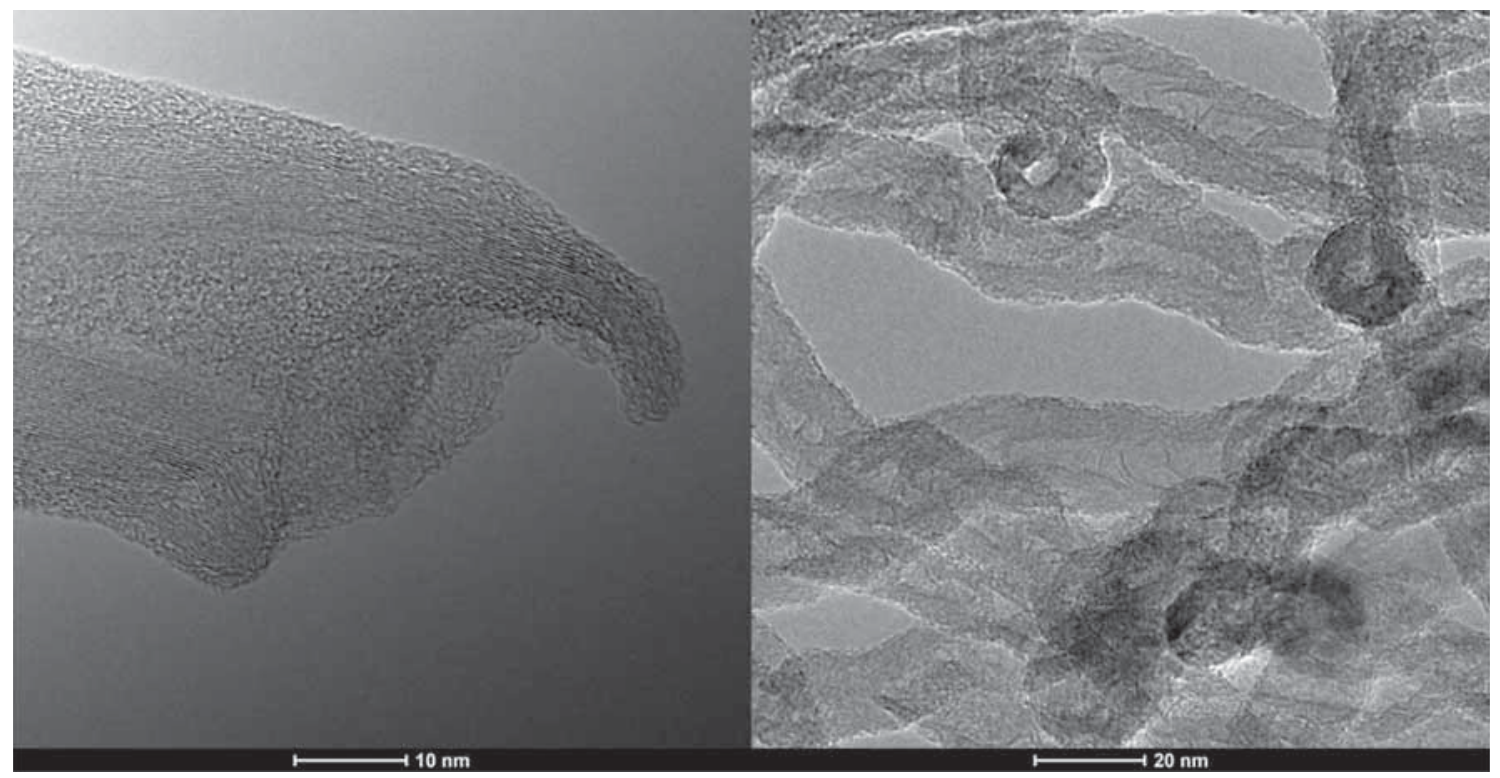

Figure 3. HR-TEM images after the MWCNTs_ld opening procedure.

$249 \mathrm{~m}^{2} \mathrm{~g}^{-1}$ for MWCNT with large diameters. In case of all the materials subjected to the adsorption of the dyes, a decrease in the total surface area was noted. This effect proved the expected (at least partial) encapsulation of the dyes. In the case of series MWCNT_ld, the maximum decrease was less spectacular i.e., the surface area decreased by $21 \mathrm{~m}^{2} \mathrm{~g}^{-1}$ in 6T@MWCNT_ld in comparison with the maximum decrease of $102 \mathrm{~m}^{2} \mathrm{~g}^{-1}$ in the 6T@MWCNT_sd sample. Thus, the OTs dyes were blocking the tube interior more effectively in MWCNTs_sd than MWCNTs_ld. The minor decrease of $S_{\mathrm{BET}}$ is understandable, provided the formation of a thin dye layer was assumed in contact with internal and external CNTs' surfaces. The thickness of the layer is too low to block the internal tube volume in MWCNTs_ld. On the contrary, the internal dye layer thickness is more comparable to the internal diameter of MWCNTs_sd. Additionally, the amount of the dyes adsorbed by the MWCNTs_ld may be smaller, since the multi-walled structure of such tubes may cause a weaker attractive forces between MWCNTs and organic compounds.

Figure 3 proves that CNTs become open-ended after the oxidation and purification procedure as depicted in figure 3 .

Figure 3 shows that the CNTs were opened and shortened significantly by oxidation, which means that dye molecules (as well as other adsorbates) can penetrate into the tube interior. Generally, the diameter of the inner tubes starts from $10 \mathrm{~nm}$. Moreover, HR-TEM observations clearly reveal that the MWCNT nanostructure exhibits the typical thick multi-walled graphitic structure covered by a small number of amorphous carbon particles. High purity and open-end structure of these CNTs are favourable for molecular encapsulation.

The application of SEM supplied with EDX microprobe allowed to obtain additional information about the chemical composition and the quality of the sample. In particular, determining the purity of CNTs is often an important component of structural and chemical characterizations for further testing of dye deposition on the surface of the examined nanotubes.

Figure 4 shows SEM images of CNTs after $\mathrm{HCl}$ treatment. MWCNTs_sd morphology is more cohesive in comparison with the MWCNTs_ld.

EDX measurements were based on the elemental analysis collected at three points of each CNT sample. Then, arithmetic average was calculated and considered as a representative value. Such mean values corresponding to particular element content are presented in table 3 (CNTs after oxidative opening and $\mathrm{HCl}$ purification) as well as in tables 4 and 5 (CNTs 

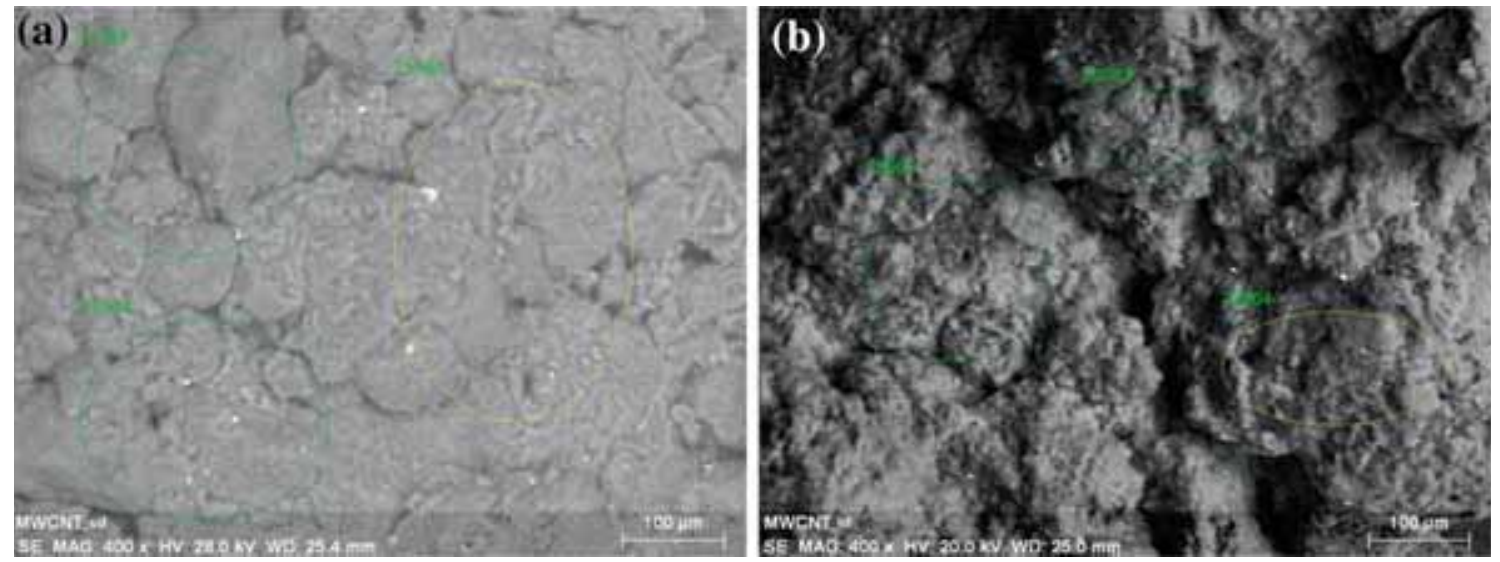

Figure 4. SEM images of (a) MWCNT_sd and (b) MWCNT_ld after the first purification.

Table 3. Elemental content of principal elements in MWCNT_sd and MWCNT_ld after the first purification (studied by SEM-EDX).

\begin{tabular}{lcccccc}
\hline & & \multicolumn{5}{c}{ Elemental content (wt\%) } \\
\cline { 3 - 7 } Sample & Domain & C & O & Mn & S & Cl \\
\hline \multirow{2}{*}{ MWCNT_sd } & 21363 & 72.65 & 26.68 & 0.30 & 0.00 & 0.37 \\
& 21364 & 72.43 & 26.86 & 0.31 & 0.00 & 0.40 \\
& 21365 & 72.12 & 27.45 & 0.18 & 0.00 & 0.25 \\
& Mean value & 72.40 & 27.00 & 0.26 & 0.00 & 0.34 \\
MWCNT_ld & 20962 & 76.39 & 22.05 & 0.82 & 0.00 & 0.74 \\
& 20963 & 76.67 & 21.59 & 0.91 & 0.00 & 0.83 \\
& 20964 & 74.64 & 24.08 & 0.54 & 0.00 & 0.74 \\
& Mean value & 75.90 & 22.57 & 0.76 & 0.00 & 0.77 \\
\hline
\end{tabular}

after the dye adsorption/encapsulation). EDX measurements (table 3) proved that sulphur was not detected, which was expected because unmodified and pure CNTs should not contain sulphur by definition. After $\mathrm{HCl}$ treatment, applied in order to eliminate $\mathrm{MnO}_{2}$ from the materials under investigation, the low Mn content (below 1\%) is within the error of the apparatus. These results show that $\mathrm{Mn}$ and $\mathrm{Cl}$ were successfully removed by intensive water rinsing. The residual content of $\mathrm{Mn}$ is related to the oxidation reaction, and in particular to $\mathrm{MnO}_{2}$, which is one of the oxidation products. A small percentage of impurities allows to start the dye encapsulation/adsorption procedure. More information and images can be found in the supporting information (table S1, figure S1a, S1b and S1c).

The elemental composition of the samples subjected to the contact with dye solution was studied by EDX method and the results are in tables 4 and 5 for MWCNT_ld and MWCNT_sd, respectively.

After dye encapsulation/adsorption, the sulphur content visibly increased proving the presence of OT in the synthesized hybrid materials. However, the sulphur content is the lowest in the case of MWCNTs_ld in comparison with the samples incorporating MWCNTs_sd (see table 5). This result is consistent with the results of surface area measurements. The surface area of MWCNTs_ld originated samples was less altered by the encapsulation/adsorption of the dyes. Summing these two effects, we let to conclude that MWCNTs_ld are inferior to MWCNTs_sd regarding their ability to adsorb the dyes under investigation.

The content of sulphur can be attributed both to the encapsulated dye molecules as well as to the ones adsorbed on the external surfaces of the CNTs of both types. In the case of dye-modified MWCNT_sd, the oxygen content is much larger relative to dye-modified MWCNTs_ld samples, suggesting that oxidation is much more considerable in MWCNTs_sd. The results of EDX also confirm that the OT dyes were better deposited onto MWCNTs_sd surface than on MWCNTs_ld. Additionally, to confirm the hypothesis on bimodal immobilization of the dyes (internal encapsulation and external layered adsorption), extensive HR-TEM studies were performed and some representative results are presented in figure 5 .

On the basis of presented HR-TEM pictures (figures 3 and 5), several observations can be made. Firstly, MWCNTs with larger diameters are long. Moreover, the external surface is not covered by loose impurities, such as catalyst residues, which confirm the effectiveness of the treatment of the samples using $\mathrm{HCl}$.

The diameters of the CNTs are directly determined from HR-TEM images with high accuracy. Thus, the MWCNTs' diameter ranges from 10 to $20 \mathrm{~nm}$. The diameter of the inner tubes is generally of the order of a few nanometres, which should allow for the adsorption and introduction of OTs into the inner surface of such MWCNTs. In addition, the tube interior is partially filled with an amorphous matter, which can be interpreted as dye molecules. Besides, the external surface is also covered by an amorphous matter that can be interpreted as adsorbed OT molecules. Similar observations were made regarding encapsulation of the dyes in MWCNTs with smaller diameters (T@MWCNTs_sd, 
Table 4. Elemental composition (by EDX) of dye-modified MWCNT_ld: T@MWCNT_ld, 3T@MWCNT_ld and 6T@MWCNT_ld after encapsulation.

\begin{tabular}{lcccccc}
\hline & & \multicolumn{5}{c}{ Elemental content (wt\%) } \\
\cline { 3 - 7 } Sample & Domain & C & O & S & Cl & Residuals \\
\hline \multirow{2}{*}{ MWCNT_ld } & 26890 & 78.68 & 20.26 & 0.00 & 0.84 & 0.22 \\
& 26891 & 78.77 & 20.13 & 0.00 & 0.87 & 0.23 \\
& 26892 & 78.46 & 20.34 & 0.00 & 0.91 & 0.29 \\
& Mean value & 78.64 & 20.24 & 0.00 & 0.87 & 0.25 \\
T@MWCNT_ld & 26896 & 78.86 & 20.21 & 0.01 & 0.71 & 0.21 \\
& 26897 & 78.25 & 20.70 & 0.04 & 0.78 & 0.23 \\
& 26898 & 78.63 & 20.31 & 0.05 & 0.83 & 0.18 \\
& Mean value & 78.58 & 20.41 & 0.03 & 0.77 & 0.21 \\
& 26899 & 76.51 & 22.21 & 0.27 & 0.81 & 0.20 \\
& 26900 & 75.75 & 23.03 & 0.26 & 0.76 & 0.20 \\
6T@MWCNT_ld & 26901 & 79.99 & 19.17 & 0.19 & 0.49 & 0.16 \\
& Mean value & 77.42 & 21.47 & 0.24 & 0.69 & 0.18 \\
& 26893 & 77.52 & 21.55 & 0.06 & 0.68 & 0.19 \\
& 26894 & 80.86 & 18.05 & 0.22 & 0.57 & 0.30 \\
& 26895 & 79.51 & 19.35 & 0.21 & 0.69 & 0.24 \\
& Mean value & 79.29 & 19.65 & 0.16 & 0.65 & 0.25 \\
\hline
\end{tabular}

Table 5. Elemental composition (by EDX) of dye-modified MWCNT_ls: T@MWCNT_1s, 3T@MWCNT_ls and 6T@MWCNT_ls after encapsulation.

\begin{tabular}{lcccccc}
\hline Sample & Domain & C & O & S & Cl & Residuals \\
\hline MWCNT_sd & 21539 & 70.78 & 26.41 & 0.00 & 0.00 & 2.81 \\
& 21540 & 70.84 & 26.45 & 0.00 & 0.00 & 2.71 \\
& 21541 & 70.56 & 27.41 & 0.00 & 0.00 & 2.03 \\
& Mean value & 70.73 & 26.76 & 0.00 & 0.00 & 2.51 \\
T@MWCNT_sd & 21542 & 69.36 & 29.23 & 0.24 & 0.66 & 0.51 \\
& 21543 & 67.48 & 30.88 & 0.29 & 0.25 & 1.10 \\
& 21544 & 69.41 & 29.05 & 0.27 & 0.66 & 0.61 \\
& Mean value & 68.75 & 29.72 & 0.27 & 0.52 & 0.74 \\
3T@MWCNT_sd & 21545 & 66.98 & 30.93 & 0.65 & 0.72 & 0.72 \\
& 21546 & 67.05 & 30.63 & 0.57 & 0.70 & 1.05 \\
& 21547 & 67.09 & 30.55 & 1.00 & 0.80 & 0.56 \\
& Mean value & 67.04 & 30.70 & 0.74 & 0.74 & 0.78 \\
6T@MWCNT_sd & 21548 & 68.16 & 29.77 & 0.78 & 0.70 & 0.59 \\
& 21549 & 68.44 & 29.54 & 0.93 & 0.78 & 0.31 \\
& 21550 & 67.27 & 29.01 & 2.52 & 0.80 & 0.40 \\
& Mean value & 67.96 & 29.44 & 1.41 & 0.76 & 0.43 \\
\hline
\end{tabular}

3T@MWCNTs_sd and 6T@MWCNTs_sd). HR-TEM evidence is given in figure $5 \mathrm{~b}$ and $\mathrm{d}$.

Numerous parameters can affect the OTs deposition on CNTs, such as: concentration of the solution, the type of solvent and particularly the nature of the adsorbed species. Although it is known that HR-TEM can directly probe the encapsulation inside MWCNTs, it is difficult to determine the detailed structure of the encapsulated OT dyes. More images can be found in the supporting information (figure S1d).

To confirm the immobilization of oligomers on the outer surface of CNTs, we performed confocal microscopy studies as an alternative characterization method. Confocal microscopy has grown in importance as a tool for mainly analysing the biological systems, but it can be applied to 

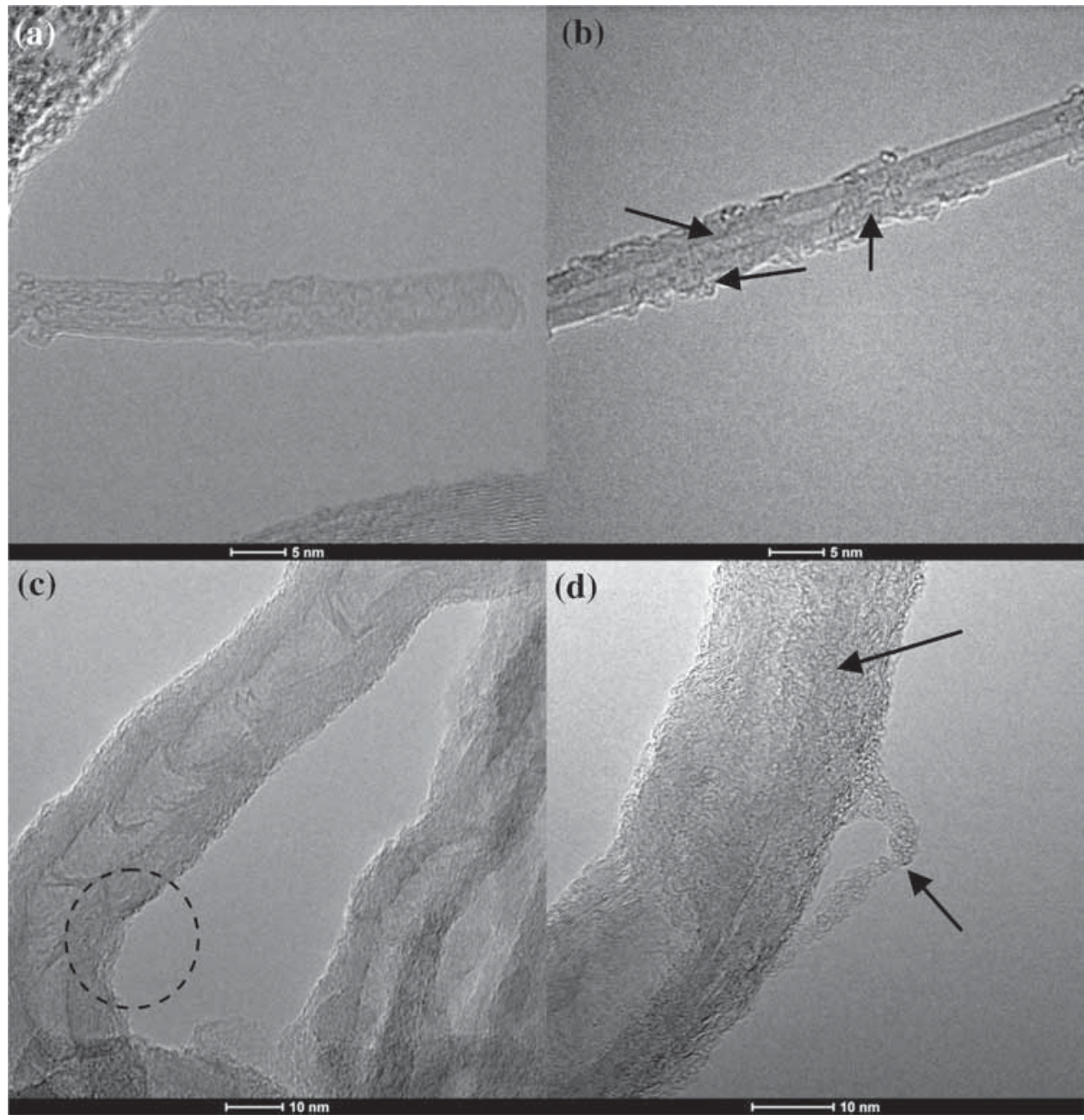

Figure 5. HR-TEM images of (a) MWCNT_sd, (b) 6T@MWCNT_sd, (c) MWCNT_ld and (d) 6T@MWCNT_ld. Arrows highlight the presence of OTs and amorphous carbon, circle indicates locations of small sidewall damage.

other materials due to high sensitivity in the detection of fluorescence [24]. Oligomers of thiophene are typically characterized by high photostability, thus, their characteristics are not significantly modified under the high excitation intensity at the focus of the objective of a fluorescence microscope [25-28]. Figure 6 presents the confocal microscopy images for3T@MWCNT_ld (blue emitting) and 6T@MWCNT_ld (red emitting). The T@MWCNT_ld sample was found to be non-fluorescent. Dark centres in figure 6 correspond to the presence of nanotubes. In turn, bright colourful areas of some representative regions indicate the presence of OT dyes. Dyes do not cover the entire surface of the nanotubes, but instead, they are clustered. The investigated OTs exhibit emission at $\lambda=441 \mathrm{~nm}\left(2,2^{\prime}: 5^{\prime}, 2^{\prime \prime}\right.$-terthiophene) and at $\lambda=594 \mathrm{~nm}$ (two vibronic side bands at 640 and $700 \mathrm{~nm}$ for $\alpha$-sexithiophene).

Confocal microscope studies showed the presence of organic dye $3 \mathrm{~T}$ and $6 \mathrm{~T}$ and their uneven distribution at the surface of MWCNTs.
Subsequently, the quality of the investigated materials was also studied by Raman spectroscopy. Raman spectroscopy has played an important role in the study and characterization of graphitic materials [29]. This method may provide information on the intermolecular interactions, which may occur in some systems like dye molecule, CNT. The Raman spectra of the tested MWCNT_ld samples are shown in figure 7a. There are differences in the intensities of the signals for various materials and the frequencies strongly depend on the nanotubes' diameter.

For the studied samples of MWCNTs_ld, the Raman spectra were measured at the excitation wavelength of $488 \mathrm{~nm}$. The most intense bands were found in the spectral region between 600 and $1600 \mathrm{~cm}^{-1}$. Furthermore, an additional peak appears at $1452 \mathrm{~cm}^{-1}$ in 6T@MWCNT_ld, which is not present in the typical spectra of pure CNTs. The two bands at 1050 (the $\mathrm{C}-\mathrm{H}$ bending) and $1452 \mathrm{~cm}^{-1}$ (the $\mathrm{C}=\mathrm{C}$ stretching) can be ascribed to the $6 \mathrm{~T}$ signals, while the bands at $\sim 1390$ 

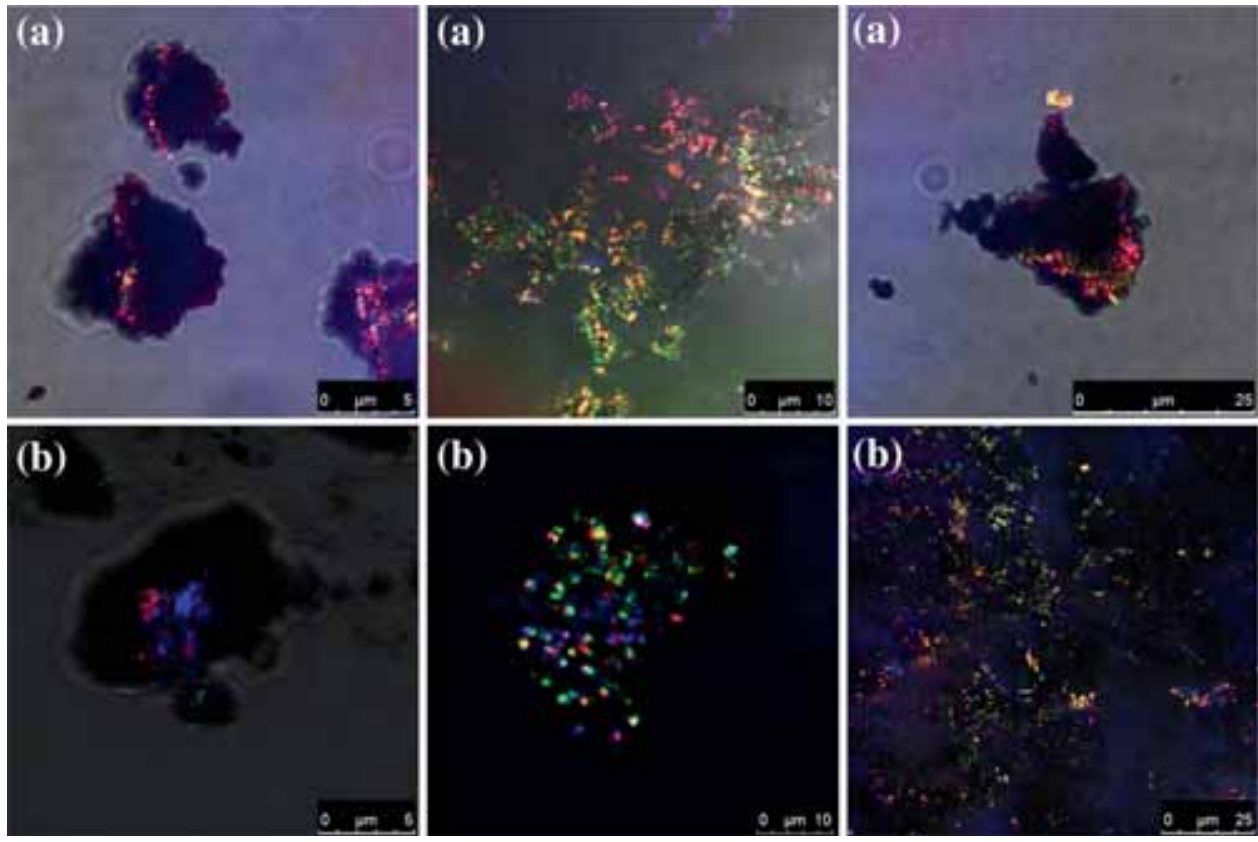

Figure 6. Confocal microscopy images for (a) 3T@MWCNT_ld sample and (b) 6T@MWCNT_ld sample.

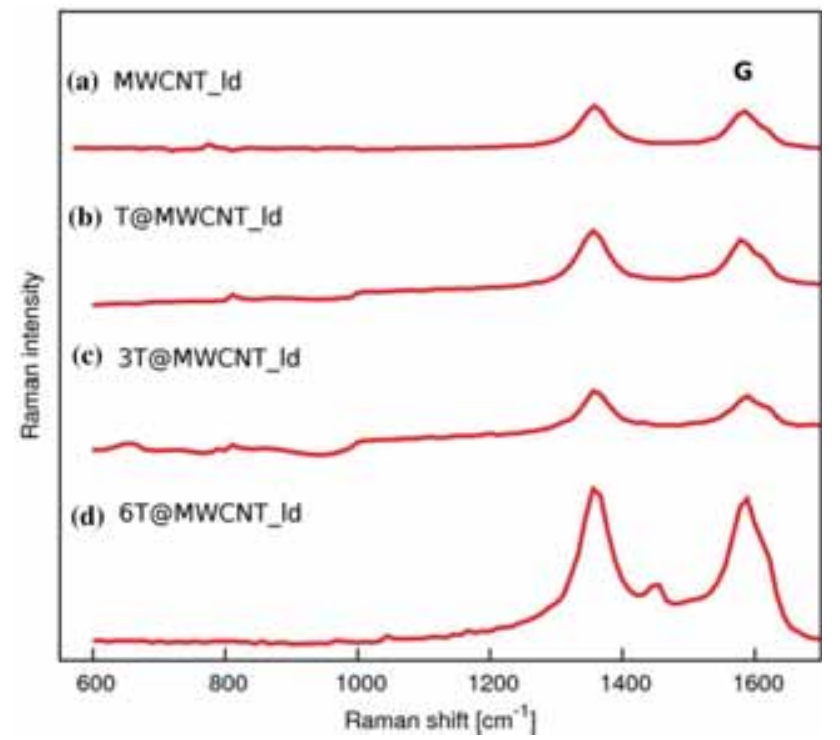

Figure 7. Raman spectra of (a) MWCNT_ld, (b) T@MWCNT_ld, (c)3T@MWCNT_ld and (d)6T@MWCNT_ld.

and $1590 \mathrm{~cm}^{-1}$ originate from nanotubes $(\mathrm{C}=\mathrm{C}$ stretching). The band at $1590 \mathrm{~cm}^{-1}$ (G band) is common to all $\mathrm{sp}^{2}$ carbon forms. These results of spectra confirm that $6 \mathrm{~T}$ adopts the endohedral position in MWCNT, which was previously reported in ref. [4] by other authors.

In figure 8, the Raman spectra measured for the MWCNTs_sd samples excited at a $532 \mathrm{~nm}$ laser line are reported. These results showed some similarities to the Raman spectra of the MWCNTs_ld samples, as apparent from figure 7.
Figure 8 clearly demonstrated the characteristics of the graphitic materials. The most intense bands were found in the spectral region between 600 and $3000 \mathrm{~cm}^{-1}$. Furthermore, a new peak appears at $1443 \mathrm{~cm}^{-1}$ after the OT encapsulation only for6T@MWCNT_sd (the C=C stretching, band derived from the dye 6T). Bands at 1390,1573 and $2667 \mathrm{~cm}^{-1}$ are characteristic of CNTs $(\mathrm{C}=\mathrm{C}$ stretching). The band around $1390 \mathrm{~cm}^{-1}$ is known as the $\mathrm{D}$ band and it suggests the presence of some small disorders or defects of the nanotube structures [29]. A band at $2667 \mathrm{~cm}^{-1}$ is called as G' band and is attributed to the overtone of the $\mathrm{D}$ band. When comparing these Raman spectra of nanotubes in all the samples, it can be concluded that the more prominent $\mathrm{D}$ band at $1390 \mathrm{~cm}^{-1}$ in MWCNTs indicates more disorder in the carbon structure.

Most applications of these new materials rely on their surface properties. Therefore, the surface elemental composition and information about the functional groups of the obtained materials were also determined by means of XPS spectra. To discuss the differences in the adsorption manner between the inner and the outer surfaces of CNTs, an additional experiment using a closed-end tube was carried out.

To prove the dye adsorption on the outer side of tubes, XPS spectra of consciously selected samples were examined to define the presence and the chemical state of sulphur, carbon and oxygen.

The examined samples were modified with $6 \mathrm{~T}$ dye and differed with regard to the way of the dye introduction. Samples6T@MWCNTs_sd and 6T@MWCNTs_ld were firstly chemically treated i.e., opened and purified. Thus, 6T dye molecules have a chance to be encapsulated and adsorbed on the outer tube surface as well. The third sample 


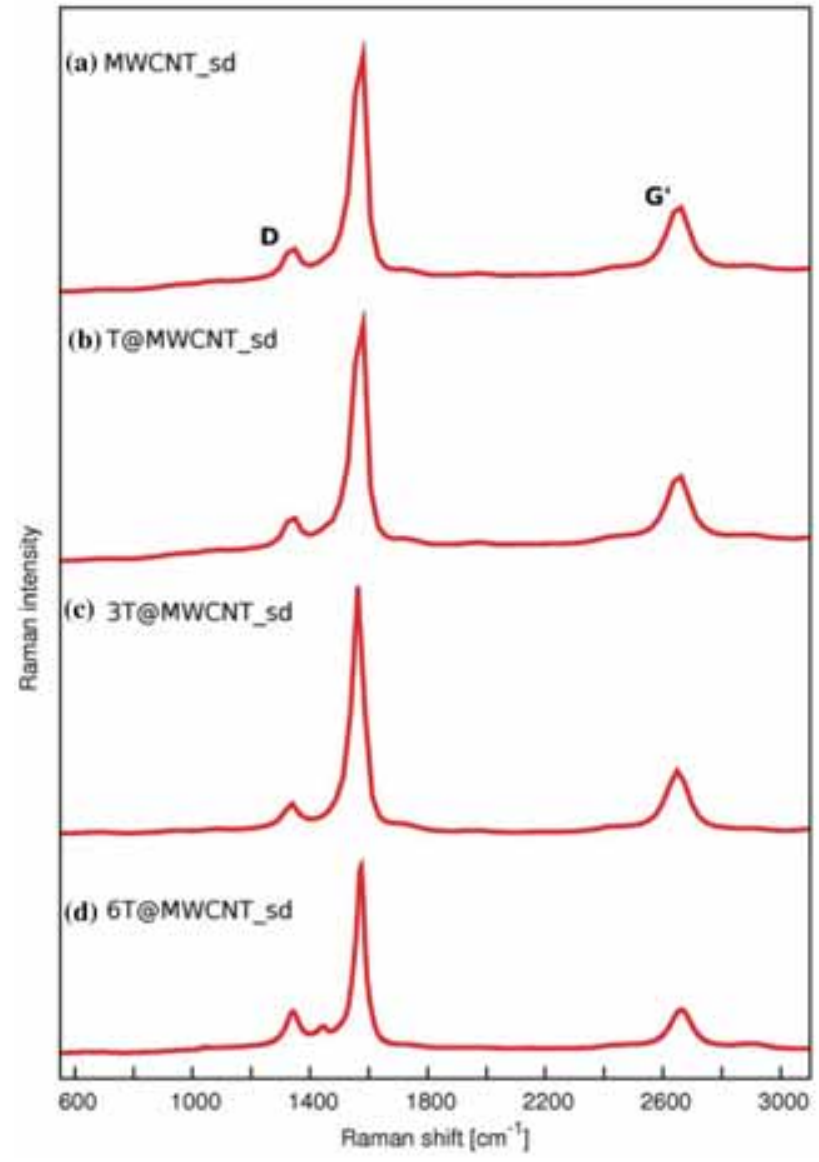

Figure 8. Raman spectra of (a) MWCNT_sd, (b) T@MWCNT_sd, (c) 3T@MWCNT_sd and (d) 6T@MWCNT_sd.

6T@MWCNTs_ld_non-functionalized (closed) was manufactured without the opening and purification procedure. Therefore, the $6 \mathrm{~T}$ dye molecules can be adsorbed by the outer surface of MWCNTs_ld and an encapsulation is excluded. Regarding the 'surface" nature of XPS method, the external 6T dye molecules are easier to be detected, if are located out of the tubes. Opened MWCNTs_sd tubes according to HR-TEM and confocal microscope studies can retain the dye molecules on the outer surface. Therefore, XPS studies also have proven the presence of sulphur. As seen from XPS investigations, also closed tubes of 6T@MWCNTs_ld_non-functionalized can retain the dyes on the outer surface in a measurable quantity. The same, but opened6T@MWCNTs_ld tubes apparently do not contain sulphur but in fact, there is no signal from 6T dye molecules adsorbed on the outer side of the tube. However, nitrogen adsorption studies and EDX measurements suggest the presence of minor amount of the dye inside the tubes, which makes them hardly observable by XPS method.

As shown in table 6, the high-resolution XPS C1s spectra confirms the existence of six (6T@MWCNTs_sd) or seven (6T@MWCNTs_ld) different carbon peaks centred at 284.5, 285.1, 286.0, 288.2, 289.3, 291.2, $293.2 \mathrm{eV}$, which can be attributed to the $\mathrm{sp}^{2} \mathrm{C}(\mathrm{C}-1), \mathrm{sp}^{3} \mathrm{C}(\mathrm{C}-2), \mathrm{C}-\mathrm{O}(\mathrm{C}-3)$,
$\mathrm{C}=\mathrm{O}(\mathrm{C}-4), \mathrm{O}=\mathrm{C}-\mathrm{O}(\mathrm{C}-5)$ and $\pi-\pi *$ shake-up features $(\mathrm{C}-6$, $\mathrm{C}-7)$, respectively. The analysis of the sulphur spectra confirms that the sulphur atoms were assigned to the two peaks centred at 163.9 and $165.0 \mathrm{eV}$. The sulphur content is very low and typically requires a long acquisition time for a good quality S2p spectrum. The high-resolution spectra of the sulphur $2 \mathrm{p}$ region shows that the thiophene ring structure is intact (figure S1e in supporting information) and detected in this form in the modified samples. The analysis of the $\mathrm{O} 1 \mathrm{~s}$ spectra suggests that oxygen is present in several possible connections. Generally, groups signed in table 6 as O-1 and O-2 are the main peaks on these spectra. A binding energy of $531.1 \mathrm{eV}(\mathrm{O}-1)$ and $532.9 \mathrm{eV}(\mathrm{O}-2)$ for these peaks is assigned for molecular oxygen and $\mathrm{C}-\mathrm{O}$ bonds, respectively. Additionally, XPS survey spectra of representative samples are shown in figure S1f of supporting information.

XRD is known as one of the most reliable techniques to check whether molecules exist inside or outside CNTs. Therefore, XRD analysis was used to observe the changes in the unmodified nanotubes and dye absorbed nanotubes. Figure 9 depicts the typical XRD pattern of the MWCNTs. The strongest diffraction peak at the angle $(2 \theta)$ of $26^{\circ}$ can be indexed as the $\mathrm{C}(002)$ reflection of the hexagonal graphite structure. The other characteristic diffraction peaks of graphite at $2 \theta$ of about 44,54 and $78^{\circ}$ are associated with $\mathrm{C}(100), \mathrm{C}(004)$ and $\mathrm{C}(110)$ diffractions of graphite, respectively. No peaks associated to the presence of crystalline oligoheterocyclic dyes were noticed. It proves that these dyes do not precipitate as excess crystalline particles between individual CNTs.

\section{Theoretical calculations}

Numerous experimental and theoretical researches show that the physicochemical properties of these new materials are highly dependent on their specific interactions [30-35]. Intermolecular interactions can significantly modify the properties of the investigated hybrid systems, which in turn have potential importance in a wide range of electronic and chemical applications. Therefore, these materials continuously remain of interest for photovoltaic materials, nanoelectronics, organic devices components and many other fields of science. To imagine how this nanotube-OTs interaction looks and whether these interactions have the power to change or modify the tubular structure, a series of preliminary models was investigated with computational chemistry tools, namely density functional theory (DFT) calculations. Theoretical calculations for the non-covalent interacting systems, where the main contribution to the interaction energy comes from the dispersion interaction - as it is a case for the CNT-require the application of modern methods of quantum chemistry. The DFT calculations can provide useful knowledge of the structure of chosen OTs inside and outside of armchair nanotubes and the interaction strength. However, hybrid systems 
Table 6. The analysis of the carbon, sulphur and oxygen contents for representative carbon materials.

\begin{tabular}{lccc}
\hline & \multicolumn{3}{c}{ Sample } \\
\cline { 2 - 4 } Peak & 6T@MWCNTs_sd & 6T@MWCNTs_ld & 6T@MWCNTs_ld_non-functionalized (closed) \\
\cline { 2 - 4 } C1s (C-1) & 44.38 & \multicolumn{2}{c}{ Content (\% mass) } \\
C1s (C-2) & 24.84 & 52.89 & 64.50 \\
C1s (C-3) & 6.46 & 14.34 & 6.72 \\
C1s (C-4) & 5.49 & 4.09 & 14.57 \\
C1s (C-5) & 3.79 & 4.15 & 3.40 \\
C1s (C-6) & 2.62 & 3.82 & 3.09 \\
C1s (C-7) & - & 1.24 & 3.45 \\
Total C & 87.58 & 88.05 & - \\
S2p3 (S-1) & 0.39 & - & 0.25 \\
S2p1 (S-2) & 0.19 & - & 0.13 \\
Total S & 0.58 & 0.00 & 0.38 \\
O1s (O-1) & 5.41 & 5.34 & 1.21 \\
O1s (O-2) & 5.63 & 6.14 & 0.37 \\
O1s (O-3) & 0.80 & 0.47 & 3.89 \\
Total O & 11.84 & 11.95 & \\
\hline
\end{tabular}

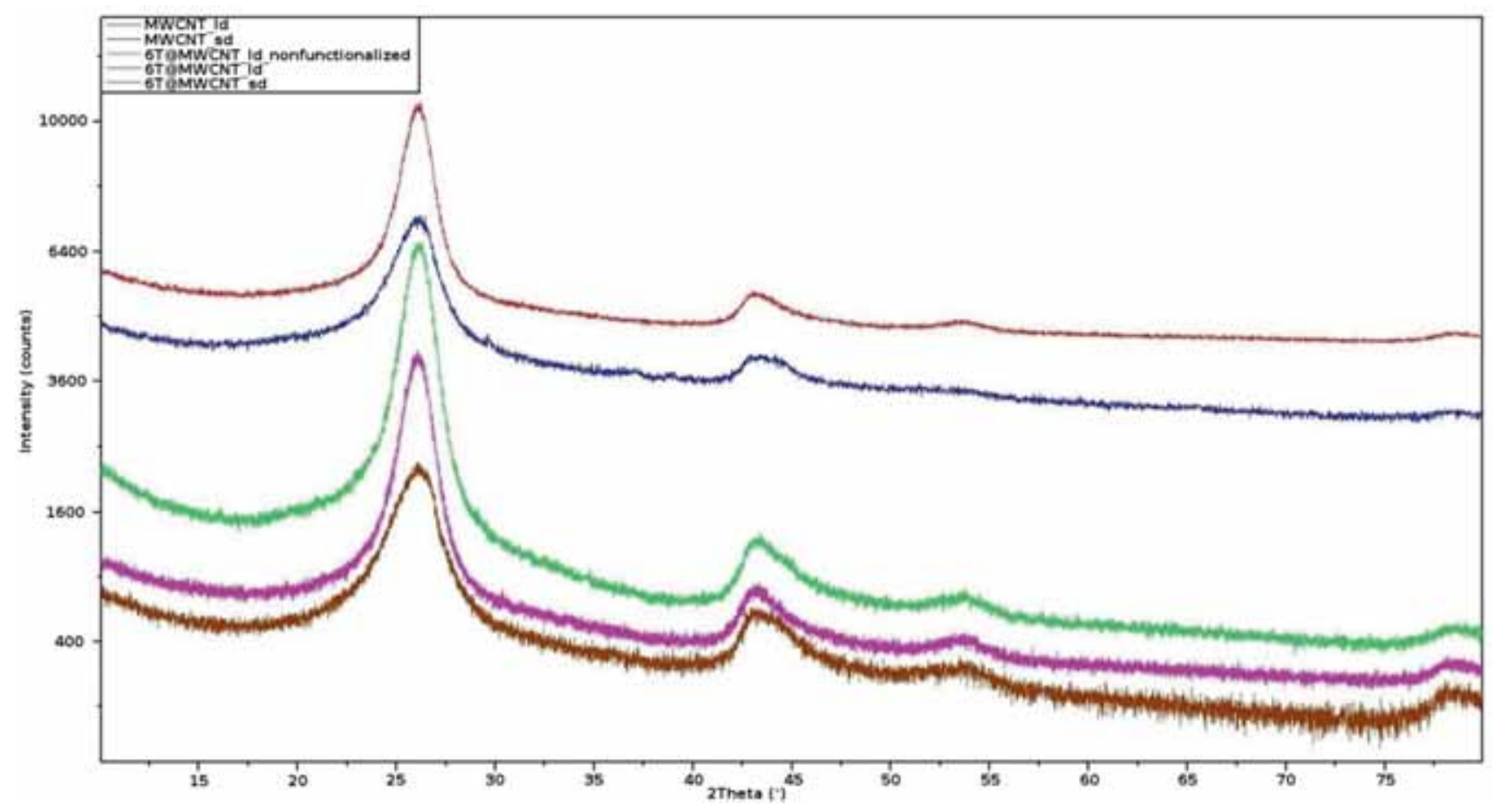

Figure 9. XRD patterns of: MWCNT_ld (red), MWCNT_sd (blue), 6T@MWCNT_ld_non-functionalized (green), 6T@MWCNT_ld (purple) and6T@MWCNT_sd (brown).

that become relevant as new promising materials are usually composed of several hundreds of atoms and therefore, calculations of their properties are too expensive. Thus, only short and small diameter models were applied here. It is known that the standard or hybrid functionals are not able to correctly reproduce the geometry of the $\pi-\pi$ interacting systems and their interaction energy [36]. Therefore, the inclusion of the Grimme dispersion corrections (functional B97D) is important to obtain the stable structures of investigated systems. The analysed hybrid systems are shown in figure 10. More images of the optimized structures are given in figure $\mathrm{S} 2 \mathrm{a}$ in supporting information. 
(a)

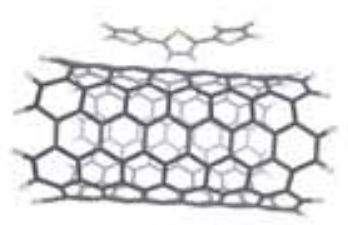

(b)

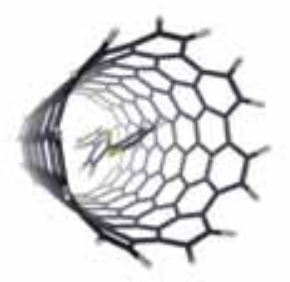

(c)

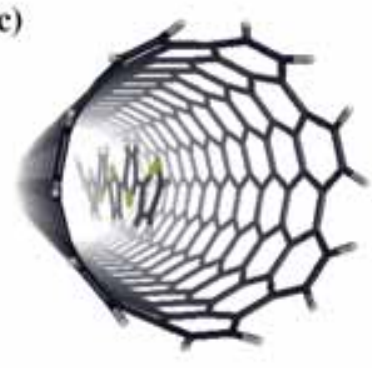

(d)

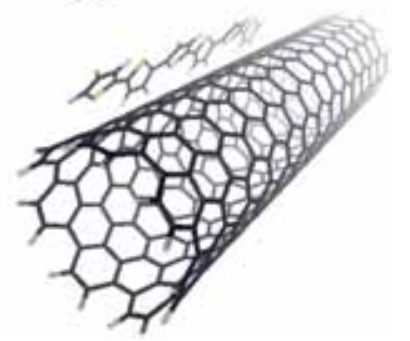

Figure 10. B97-D/6-31G(d,p) optimized structures of the representative complexes of short tube model of SWCNT with 3T (a) outside or (b) inside the tube and long tube model of SWCNT with $6 \mathrm{~T}$ (c) inside or (d) outside the tube.

The calculated interaction energy depends strongly on the orientation of the oligomer as shown in table 7. In $\pi$-electron systems, such as in OTs and CNTs, the main role is played by the dispersion interaction. The OT dyes would exhibit $\pi-\pi$ interactions with the tube wall and these interactions could shift OT molecules off the SWCNTs centre as shown in the case of SQ molecules [9]. 3T arranged inside the short nanotube have larger (more negative) interaction energies than the 3T located on the outside of the tube (table 7). Additionally, to verify the quality of the results obtained with the short tube model, the calculations were also carried out for larger tubes with a diameter of $1.3 \mathrm{~nm}$, including the adsorbed T, 3T or $6 \mathrm{~T}$ molecules.

According to the geometry optimization with the B97-D/6$31 \mathrm{G}(\mathrm{d}, \mathrm{p})$ approach, external thiophene oligomers prefer to be located near the walls of the armchair nanotubes, while internal oligomers prefer to be located at the centre of nanotubes due to the small diameter of the investigated tubes. Based on figure 10, it can be concluded that the thiophene oligomers inside and outside the nanotube tend to form nearly planar structures.

The interaction energy increases with the length of the linear OT chain. Based on theoretical calculations, it is considered that greater aromatic molecules interact strongly with SWCNTs. We assume that the van der Waals interaction, especially $\pi-\pi$ interaction between the dye and nanotube wall, would be a major force to stabilize OT dyes inside the nanotube. Eventually, the high polarizability of the sulphur atom in OT rings leads to the stabilization of the conjugated chain [37]. Sulphur atoms can also be the potential sites of interactions with a nanotube. The interaction energy of the $6 \mathrm{~T}$ molecule inside the tube walls is dominant, which suggests that this is the most stable form of the dyes bonding (encapsulation). However, the interaction energy between the dyes and the outer surface is not zero. Thus, the dye adsorption on the outer surface SWCNTs is also probable. We assume that such conclusion driven from the theoretical studies on SWCNTs may be extended to other CNTs like MWCNT_sd and MWCNTs_ld.

In further studies, additional analogous calculations will be carried out for semiconducting nanotubes, since the interaction energy between the host SWCNT and the encapsulated molecules may be highly dependent on the electric properties of the host SWCNT [38-40].

\section{Conclusions}

The interaction of OTs with CNTs were investigated by means of various experimental (nitrogen adsorption, Raman spectroscopy, XRD, SEM-EDX, HRTEM and confocal microscopy) and computational techniques. Basically, two types of

Table 7. Interaction energy of $\mathrm{T}, 3 \mathrm{~T}$ and $6 \mathrm{~T} / \mathrm{SWCNT}(7,7)$ complex in different positions calculated by using several computational methods.

\begin{tabular}{lccc}
\hline & \multicolumn{3}{c}{ Calculated interaction energy $\left(\mathrm{kcal} \mathrm{mol}^{-1}\right)$} \\
\cline { 2 - 4 } Sample & B97-D/6-31G(d,p) & B97-D3/6-31G(d,p) & WB97x-D/6-31G(d,p) \\
\hline Long tube model & & & \\
T (in) @SWCNT & -22.43 & -26.05 & -21.13 \\
T (out) @SWCNT & -7.02 & -8.66 & -7.25 \\
3T (in) @SWCNT & -57.47 & -68.12 & -55.19 \\
3T (out) @SWCNT & -20.64 & -25.15 & -20.88 \\
6T (in) @SWCNT & -114.35 & -133.79 & -40.22 \\
6T (out) @SWCNT & -40.30 & -49.12 & -53.36 \\
Short tube model & & & -19.76 \\
3T (in) @SWCNT & -55.92 & -66.69 & -24.18 \\
3T (out) @SWCNT & -19.51 & - & \\
\hline
\end{tabular}


CNTs were investigated: small diameter MWCNTs_sd (ca. $1.8-10.1 \mathrm{~nm}$ ) and large diameter MWCNTs_ld (ca. 11.2$22.5 \mathrm{~nm}$ ). The tubes were opened (wet oxidant method) and purified. All the analyses allowed to characterize the structure of the obtained CNT-OTs complexes and to determine the mechanism of the dye binding to CNTs. OT molecules were always encapsulated in both types of nanotubes, but not entirely. The potential of adsorption inside the CNTs is always greater than the outside (proved by computational approach), but in case of the adsorption of T, 3T and 6T, a mixed binding at the external-internal surfaces takes place. In some cases, a slight dominance of external binding is observed (particularly small diameter MWCNTs_sd). In case of the later type of tubes, the OT dye encapsulation leads to a visible blocking of the interior tube. This effect is hardly visible in case of large diameter tubes MWCNTs_ld. Generally, MWCNTs_ld are less effective in binding (by encapsulation and external adsorption) the OT dye as proven by EDX and XPS studies. Formation of excess dye crystals among CNTs was not detected (XRD, Raman spectroscopy), and the method of organic compound encapsulation does not lead to a lot of structural changes, while maintaining the desired properties of the carbon material. The idea was supported by a theoretical calculation. The DFT calculations were consistent with the experimental findings and let to predict the stability of such systems (the interaction energy is relatively high and more negative from $\mathrm{T}$ to $6 \mathrm{~T}$ ). $\pi$-Stacking at the outer surface of the tube leads to a strong interaction with different nanotubes.

The research and analysis presented in this article establish a first step to the thorough investigation and modification of the optical and spectral properties of various types of carbon materials, including CNTs. This aspect of the performed studies will be continued in further studies.

\section{Acknowledgements}

This research was supported in part by PL-Grid Infrastructure. Wroclaw Networking and Supercomputing Centre, Academic Computer Centre in Gdansk (CI TASK), Poznan Supercomputing and Networking Center (PCSS) and AGH Cyfronet Krakow are gratefully acknowledged for the generous allotment of computational resources.

\section{References}

[1] Di Crescenzo A, Ettorre V and Fontana A 2014 Beilstein J. Nanotechnol. 51675

[2] Bilalis P, Katsigiannopoulos D, Avgeropoulos A and Sakellariou G 2014 RSC Adv. 42911

[3] Colomer J F, Piedigrosso P, Fonseca A and Nagy J B 1999 Synth. Met. 1032482

[4] Loi M A, Gao J, Cordella F, Blondeau P, Menna E, Bartova B et al 2010 Adv. Mater. 221635
[5] Giulianini M, Waclawik E R, Bell J M, De Crescenzi M, Castrucci P, Scarselli M et al 2009 Appl. Phys. Lett. 95 013304

[6] Simon F, Peterlik H, Pfeiffer R, Bernardi J and Kuzmany H 2007 Chem. Phys. Lett. 445288

[7] Fan J, Yudasaka M, Yuge R, Futaba D N, Hata K and Iijima S 2007 Carbon $\mathbf{4 5} 722$

[8] Al Garalleh H, Thamwattana N, Cox B J and Hill J M 2016 J. Biomater. Tissue Eng. 6362

[9] Yanagi K, Iakoubovskii K, Matsui H, Matsuzaki H, Okamoto H, Miyata Y et al 2007 J. Am. Chem. Soc. 1294992

[10] Zhang L, Colella N S, Cherniawski B P, Mannsfeld S C B and Briseno A L 2014 ACS Appl. Mater. Interf. 65327

[11] Costa J C S, Taveira R J S, Lima C F R A C, Mendes A and Santos L M N B F 2016 Opt. Mater. 5851

[12] Wilbers J G E, Xu B, Bobbert P A, de Jong M P and van der Wiel W G 2017 Sci. Rep. 741171

[13] Gidron O, Varsano N, Shimon L J W, Leitus G and Bendikov M 2013 Chem. Commun. 496256

[14] Gao W, Xu W, Ye J, Liu T, Wang J, Tan H et al 2017 Adv. Funct. Mater. $\mathbf{4 2} 1703938$

[15] Lima C F R A C, Costa J C S, Galvao T L P, Tavares H R, Silva A M S and Santos L M N B F 2014 Phys. Chem. Chem. Phys. 1614761

[16] Skotheim T A and Reynold J R 2007 Handbook of conducting polymers (United States: CRC Press)

[17] Saini G, Lucas N T and Jacob J 2010 Tetrahedron Lett. 51 2956

[18] Iijima S 2002 Physica B 3231

[19] Galano A 2006 Chem. Phys. 327159

[20] Tsuzuki S, Honda K and Azumi R 2002 J. Am. Chem. Soc. 124 12200

[21] Frisch M J, Trucks G W, Schlegel H B, Scuseria G E, Robb M A, Cheeseman J R et al 2009 Gaussian 09, Revision D.01, Gaussian Inc., Wallingford CT

[22] Schaftenaar G and Noordik J H 2000 J. Comput.-Aid Mol. Des. 123123

[23] Balasubramanian K and Burghard M 2005 Small 1180

[24] Tabor R F, Lockie H, Mair D, Manica R, Chan D Y C, Grieser F et al 2011 J. Phys. Chem. Lett. 2961

[25] Hartschuh A, Pedrosa H N, Peterson J, Huang L, Anger P, Qian H et al 2005 Chem. Phys. Chem. 6577

[26] Alvarez L, Almadori Y, Mariot S, Aznar R, Arenal R, Michel T et al 2013 J. Nanoelectron. Optoelectron. 828

[27] Fichou D and Charra F 1996 Synth. Met. 7611

[28] Capobianco M L, Barbarella G and Manetto A 2012 Molecules 17910

[29] Hodkiewicz J and Wall M 2010 NSTI-Nanotech. 1318

[30] Moreno-Castilla C 2004 Carbon 4283

[31] McIntosh G C, Tomanek D and Park Y W 2003 Phys. Rev. B 67125419

[32] Koh B, Kim G, Yoon H K, Park J B, Kopelman R and Cheng W 2012 Langmuir 2811676

[33] Anota E C, Cocoletzi G H and Tapia A M G 2015 Open Chem. 13734

[34] Singh-Miller N E, Scherlis D A and Marzari N 2006 J. Phys. Chem. B 11024822

[35] Schuth F 2014 Chem. Mater. 26423

[36] Kaczmarek-Kedziera A, Ziegler-Borowska M and Kedziera D 2014 Chemia obliczeniowaw laboratorium organicznym (Poland: Wydawnictwo Naukowe UMK Torun) 
[37] Perepichka I F and Perepichka D F 2009 Handbook of thiophene-based materials: applications in organic electronics and photonics (United States: John Wiley \& Sons Ltd)

[38] Lehman J H, Terrones M, Mansfield E, Hurst K E and Meunier V 2011 Carbon 492581
[39] Grazioli C, Baseggio O, Stener M, Fronzoni G, de Simone M, Coreno M et al 2017 J. Chem. Phys. 146 054303

[40] Yoshida Y, Ishii Y, Kato N, Li C and Kawasaki S 2016 J. Phys. Chem. C 12020454 